\title{
Multi-view representation learning and understanding
}

Published online: 14 January 2021

(C) Springer Science+Business Media, LLC, part of Springer Nature 2021

Multimedia Tools and Applications gratefully acknowledges the editorial work of the scholars listed below on the special issue entitled "Multi-view Representation Learning and Understanding" (SI $1142 \mathrm{~T}$ ).

Of 47 papers submitted to this issue, 14 were eventually accepted after a stringent peer review process.

\section{Corresponding Guest Editor}

\section{Tao Zhou}

University of North Carolina at Chapel Hill, USA

Email: taozhou@email.unc.edu

\section{Guest Editors}

Yu Zhang

Stanford University, USA

Email: yzhangsu@stanford.edu

\section{Kim-Han Thung}

University of North Carolina at Chapel Hill, USA

Email: khthung@med.unc.edu

Ehsan Adeli

Stanford University, USA

Email: eadeli@stanford.edu

\section{Islem Rekik}

University of Dundee, UK

Email: irekik@dundee.ac.uk

\section{Qibin Zhao}

RIKEN AIP, Japan

Email: qbzhao@brain.riken.jp

\section{Changqing Zhang}

Tianjin University, China

Email: zhangchangqing@tju.edu.cn

Publisher's note Springer Nature remains neutral with regard to jurisdictional claims in published maps and institutional affiliations. 